\title{
Estrategia de gestión de la energía en vehículos eléctricos con pila de combustible y sistema de almacenamiento híbrido utilizando control predictivo económico Energy management strategy for electric vehicles with
hybrid storage system using economic predictive
control
}

José Luis Sampietro, Vicenç Puig and Ramon Costa Castelló

\begin{abstract}
In this work, economic predictive control is proposed as a technique for optimum energy management of a hybrid vehicle. The model used for the control is described and an economic predictive controller is designed from it. Finally, the best adjustment of the weights of the controller that achieves the greatest reduction in fuel cell consumption with the help of batteries and supercapacitors is studied. For this purpose, the maximum and minimum consumption points of the fuel cell are determined, and the multiobjective control problem is characterized through the determination of the Pareto curves. The article concludes with a discussion of the results and future work.
\end{abstract}

Index Terms-Economic predictive control, hybrid vehicles, fuel cell, batteries, supercapacitors, multi-objective control

Resumen-En este trabajo se propone el control predictivo económico como técnica para la gestión energética óptima de un vehículo híbrido. Se describe el modelo utilizado para el control y a partir del mismo se diseña un controlador predictivo económico. Finalmente, se estudia el mejor ajuste de los pesos del controlador que consiga la mayor reducción del consumo de la pila de combustible con la ayuda de baterías y supercapacitores. Para ello se determinan los puntos de consumo máximo y mínimo de la pila de combustible, y se caracteriza el problema de control multiobjetivo mediante la determinación de las curvas de Pareto. El artículo concluye con una discusión de los resultados y los trabajos futuros.

Palabras Claves-Control predictivo económico, vehículos híbridos, pila de combustible, baterías, supercapacitores, control multiobjetivo

José Luis Sampietro pertenece a la Facultad de Ingenierías de la Universidad Técnica Luis Vargas Torres, Esmeraldas, Ecuador (email: jose.sampietro@utelvt.edu.ec).

Vicenç Puig y Ramon Costa Castelló pertenecen al Departamento de Ingeniería de Sistemas, Automática e Informática Industrial (ESAII), Barcelona, España (email: vicenc.puig@upc.edu, ramon.costa@upc.edu).

\section{INTRODUCTION}

$\mathrm{S}$ EGúN [1], como consecuencia de la utilización de vehículos con motores convencionales de combustión interna, el consumo de gasolina en el sector del transporte ha aumentado a un ritmo superior al de cualquier otro sector en las últimas décadas. Algunos problemas medioambientales están directamente relacionados con las emisiones de los vehículos, como por ejemplo el efecto invernadero, la lluvia ácida o el smog fotoquímico. Tomando en consideración que los productos derivados del petróleo, son no renovables, como se lo menciona en [2], y dado que las actividades de los seres humanos contribuyen al agravamiento del cambio climático, es importante cambiar el comportamiento mencionado. En [3], menciona que el cambio a tecnologías que contemplen vehículos que funcionen con sistemas híbridos (HEV) y eléctricos $(\mathrm{EV})$ para el transporte personal y comercial puede ser una manera adecuada.

Contrariamente a los vehículos de motores de combustión interna, el vehículo eléctrico híbrido ayuda a reducir las emisiones de carbono. De acuerdo con [4]-[7] si comparamos entre el vehículo eléctrico híbrido con motor de combustión y el puramente eléctrico, se puede mostrar que el último no produce emisiones. Actualmente se estudian sistemas híbridos, cuya fuente principal es una pila de combustible, en reemplazo del motor tradicional de combustión. En primera instancia se migro del motor de combustión interna solo, hacia los puramente eléctricos, con variantes como el híbrido con motor de combustión y baterías eléctricas. No obstante, la problemática de la autonomía de los vehículos puramente eléctricos, y la voluntad de reemplazar por completo el motor de combustión interna, proyecta el crecimiento del mercado hacia los vehículos híbridos de pila de combustible. Esto, debido a que se puede llevar el tanque de combustible (hidrógeno) en el vehículo y re-cargarlo como si fuese gasolina o diésel. Aunque los costes del vehículo híbrido con pila de combustible son aún elevados, varios estudios como 
los desarrollados por Ballard, indican que para el 2030 el precio podría llegar a ser competitivo.

Según [8], los vehículos alimentados por una pila de combustible, en comparación con el motor de combustión interna (siglas en inglés ICE), tienen las ventajas de una alta eficiencia energética. Para este trabajo en particular, usaremos un vehículo cuya fuente principal de energía es una pila de combustible y tiene elementos que son capaces de almacenar energía. El objetivo será gestionar de manera óptima la energía del HEV. Para esto se considera la energía que cada elemento es capaz de aportar al sistema. Se tomará un perfil de velocidad conocido, y mediante las ecuaciones correspondientes a la cinemática del vehículo se lo convertirá en un perfil de potencia. Este perfil, actuará como la demanda del sistema en donde, las fuentes deben realizar el intercambio energético, para cumplirlo. Se busca entonces que cada fuente entregue la cantidad óptima de energía (acotada) para permitir el desplazamiento, tomando en cuenta todas sus limitaciones físicas. En [9], se menciona de manera general el estado de la tecnología para cada uno de los componentes mencionados. Existen diversos trabajos que toman en consideración como fuentes de almacenamiento, ya sean baterías o supercapacitores, no obstante, la combinación de ambos ayuda a una mejor gestión energética, pues como se puede ver en [10]-[15]. Las baterías poseen una alta densidad de energía, con una dinámica lenta, mientras los supercapacitores, poseen una dinámica más rápida, lo que los hace un complemento eficiente. Entonces, el principal objetivo es que la combinación de una fuente, o varias fuentes de almacenamiento de energía reversible, con una pila de combustible permita reducir el consumo. Esto, debido a que se podrá recuperar y almacenar parte de la energía generada en el momento del frenado mediante el concepto de freno regenerativo, según las capacidades de almacenamiento de cada elemento. De la misma manera, el incluir un sistema de absorción energética como las baterías y supercapacitores, permitirá reducir el tamaño de la pila de combustible, y por ende el coste de la misma.

A partir del modelo propuesto para los elementos, se diseña un controlador acorde a los objetivos de control. En la literatura, se han estudiado muchos tipos o técnicas de control para abordar el problema mencionado, como por ejemplo programación dinámica (DP, siglas en ingles) en [16]-[18]. Control en tiempo real, con la estrategia de minimización de consumo equivalente (ECMS, siglas en inglés) como en [19]-[21]. Además de control predictivo basado en modelos (MPC, siglas en inglés), control adaptativo y control difuso como en [22]-[25]. Para el presente trabajo, usaremos un controlador predictivo económico (EMPC, siglas en inglés), como una variación del MPC tradicional. Para esto, una alternativa propuesta es colocar un objetivo económico relacionado con los costes de generación de energía directamente como parte de la función del objetivo del controlador MPC. De esta forma se optimiza directamente en tiempo real el desempeño económico del sistema, en lugar de forzar el seguimiento de un valor de consigna. Luego, en base a los puntos de consumo máximo y mínimo de la pila de combustible, se mostrará el máximo ahorro alcanzado en el sistema y se caracterizará el problema de control multiobjetivo mediante la determinación de las curvas de Pareto. El artículo concluye con una discusión de los resultados y los trabajos futuros.

La estructura del artículo es la siguiente: En la Sección 2, se presenta el modelo del vehículo utilizado para la gestión de la energía. En la Sección 3, se introduce el control predictivo económico (EMPC) utilizado para la gestión óptima de la energía. En la Sección 4, se describen los resultados de aplicación del mismo en un caso de estudio. Finalmente, en la Sección 5 se presentan las conclusiones y los trabajos futuros.

\section{Modelo Orientado Al CONTROL}

El modelo orientado a control se puede construir a partir de identificar los modelos de cada uno de los componentes del mismo: elementos de almacenamiento, bus de energía, fuentes y demandas. En este artículo, el vehículo considerado presenta los elementos que se muestran en la Fig. 1. La fuente de energía principal en el HEV considerado es una pila de combustible, y los elementos de almacenamiento de energía son una batería y un supercapacitor. Los supercapacitores y baterías, entregan energía en instantes de tiempo en los que la pila no puede cumplir con la demanda generada por el perfil, y son capaces de recuperar energía que no se disipa (en la desaceleración del perfil). Todos los elementos entregan la energía mediante un bus común, conectado a una maquina eléctrica que se conecta al sistema de tracción del vehículo.

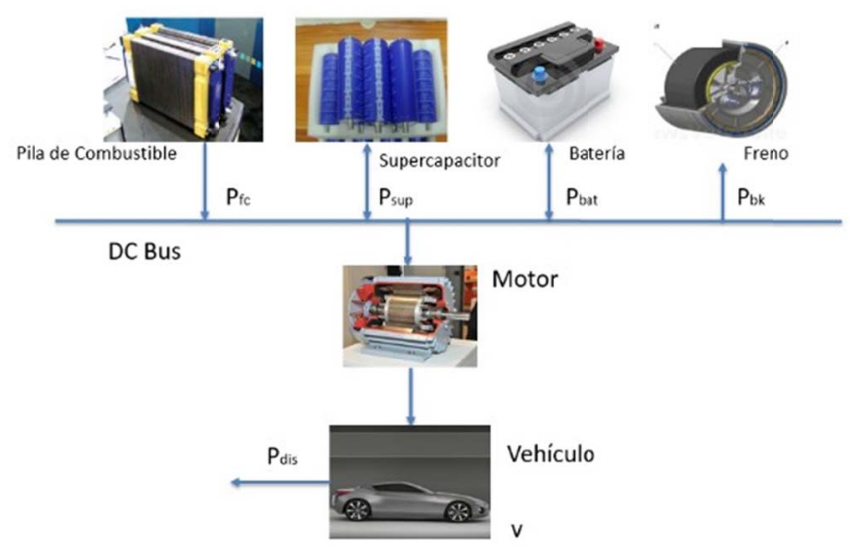

Fig. 1. Componentes del vehículo.

A continuación, se describirá el modelo orientado a control de cada uno de dichos componentes.

\section{A. Elementos de almacenamiento}

1) Modelo del supercapacitor: Los modelos más comunes para este tipo de elementos son los eléctricos, en donde tenemos una capacitancia y un resistor en serie. En [26], se realiza un estudio más en detalle de sus características y comportamiento. Una propiedad deseable de estos, es su alta densidad de potencia, es decir entregan de forma casi instantánea toda la energía que almacenan. Para el presente 
trabajo, aproximaremos un modelo energético, que será el descrito por:

$$
E_{\text {sup }}=E_{\text {sup }, 0}+\int_{0}^{t} P_{\text {sup }} d t
$$

donde $E_{\text {sup }}$ es la energía acumulada en el supercapacitor, $E_{\text {sup }, 0}$ es el valor inicial de la misma y $P_{\text {sup }}$ es la potencia neta proporcionada al supercapacitor.

El parámetro $P_{\text {sup }}$ a la vez, tendrá límites máximos de carga y descarga, teniendo en cuenta la capacidad nominal máxima de entrega de potencia $P_{\max }$. Los parámetros usados para el supercapacitor considerado se muestran en la Tabla I.

TABLA I

PARÁMETROS USADOS PARA EL SUPERCAPACITOR

\begin{tabular}{lcc}
\hline \hline Nombre & Valor & Unidad \\
\hline Voltaje Nominal & 48.6 & $\mathrm{~V}$ \\
Potencia Máxima & 60 & $\mathrm{~kW}$ \\
Capacitancia (C) & 165 & $\mathrm{~F}$ \\
\hline \hline
\end{tabular}

2) Modelo de la batería: En [27], se estudia las propiedades de las baterías, y su vida útil en función a los parámetros de carga y descarga de las mismas en aplicaciones de vehículos. El modelo energético considerado para la batería es análogo al del supercapacitor, y queda representado por:

$$
E_{b a t}=E_{b a t, 0}+\int_{0}^{t} P_{b a t} d t
$$

donde $E_{b a t}$ es la energía acumulada en la batería, $E_{b a t, 0}$ es el valor inicial de la misma y $P_{b a t}$ es la potencia neta proporcionada por la batería. El estado de carga (SOC) relaciona la cantidad de carga de una batería en relación con su capacidad nominal. En términos de energía del sistema, se puede definir como:

$$
S O C_{b a t}=\frac{E_{b a t}}{E_{b a t}^{\max }}
$$

En el caso del presente trabajo, tanto para la batería como para el supercapacitor, el valor de SOC inicial y final son iguales. Con esto se asegura que toda la energía que se recibe del freno regenerativo, sea entregada. Una manera de verificar que los elementos de almacenamiento no reciben potencia de la pila de combustible, es verificar que no exista potencia de salida en los mismos cuando el vehículo está parado. Además, que la energía recuperada sea la misma que entregan estos elementos. La energía que no pueda ser recuperada porque los elementos están con su carga máxima, se disipara en el freno mecánico. Los parámetros usados para la batería considerada se muestran en la Tabla II.

\section{B. Pila de Combustible:}

Las pilas de combustible presentan un comportamiento no lineal en su característica tensión-corriente. En estado estacionario, el modelo eléctrico más simple que se puede construir consiste en una fuente de voltaje Thevenin $V_{c}$ en serie con una resistencia $R_{c}$ cuyos valores están en función de variables como la presión de combustible, la humedad, y el estado del catalizador, como se analiza en [28]-[30].

TABLA II

PARÁMETROS USADOS PARA LA BATERÍA

\begin{tabular}{lcc}
\hline \hline \multicolumn{1}{c}{ Nombre } & Valor & Unidad \\
\hline Capacidad & 2.5 & $\mathrm{Ah}$ \\
Potencia Máxima & 80 & $\mathrm{~kW}$ \\
Máximo de carga continua & 6.5 & $\mathrm{~A}$ \\
Máximo de descarga continua & 25 & $\mathrm{~A}$ \\
\hline \hline
\end{tabular}

Es posible utilizar un modelo linealizado capaz de predecir el comportamiento de la pila, a efectos de control de la misma, quedando definido por:

$$
E_{f c}=E_{f c, 0}+\int_{0}^{t} P_{f c} d t
$$

donde $E_{f c}$ es la energía generada por la pila, $E_{f c, 0}$ es el valor inicial de energía de la pila, $P_{f_{c}}$ es la potencia neta proporcionada por la pila al movimiento y $P_{f_{c}}{ }^{\max }$ es la potencia máxima. En un sistema convencional todas las celdas de la pila están conectadas en serie, por lo que la corriente de carga en cada célula tiene que limitarse a la corriente máxima que una celda del sistema puede suministrar. En el presente trabajo, la potencia de salida será limitada entre cero y el valor de potencia máxima que puede aportar.

$$
0 \leq P_{f c} \leq P_{f c}^{\max }
$$

\section{Potencia demandada}

Se trabajará con un perfil de velocidad conocido, el mismo que para el presente trabajo será el Nuevo Ciclo de Conducción Europeo (NEDC, siglas en inglés), cuya velocidad se muestra en la Fig. 2.

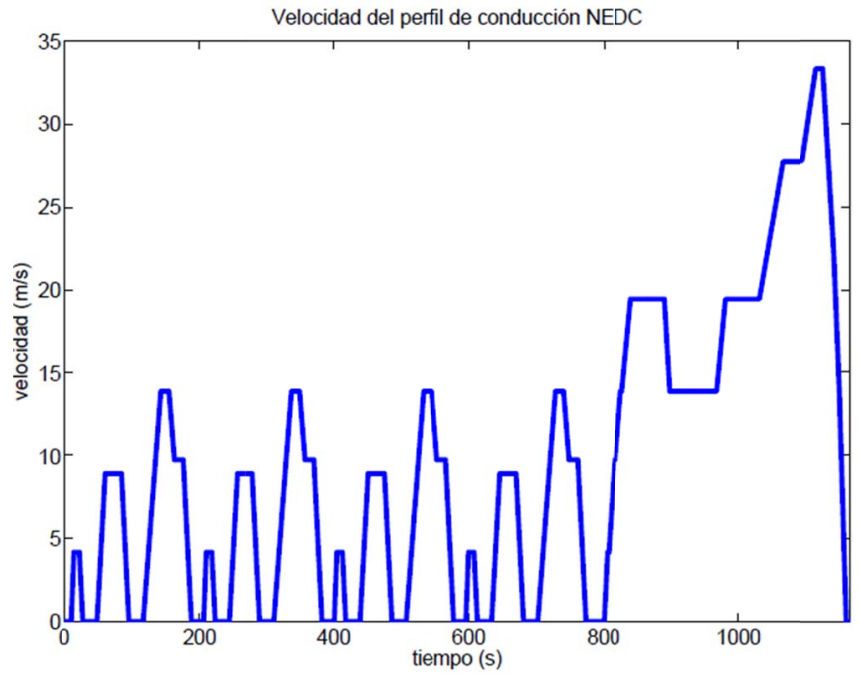

Fig. 2. Perfil de conducción NEDC.

En [31] se indica que en Europa se usa el ciclo NEDC, el cual es el criterio a seguir para homologar vehículos para su comercialización. Los ciclos de conducción intentan emular el comportamiento al volante de los usuarios, tanto en tramos 
urbanos como en interurbanos a través de una combinación de distintas velocidades en una escala temporal. Este perfil, es el que se le proporciona al controlador EMPC como una demanda a seguir, cuyos datos relevantes se encuentran en la Tabla III.

TABLA III

PERFIL DE CONDUCCIÓN NEDC

\begin{tabular}{lc}
\hline \hline \multicolumn{1}{c}{ Parámetro } & Valor \\
\hline Distancia & $11007[\mathrm{~m}]$ \\
Velocidad Media & $9,3[\mathrm{~m} / \mathrm{s}]$ \\
Velocidad máxima & $33,3[\mathrm{~m} / \mathrm{s}]$ \\
Aceleración máxima & $1,04\left[\mathrm{~m} / \mathrm{s}^{2}\right]$ \\
\hline \hline
\end{tabular}

Existen varios estudios que muestran el cálculo de la potencia demandada por un vehículo como por ejemplo [32]-[34].

Al tomar en consideración los mismos, en el caso de este artículo, la potencia mecánica necesaria para el movimiento del vehículo, que se denominará $p_{m o v}$ se calcula de la suma de la potencia cinética $p_{c}$ y la potencia necesaria para vencer las fuerzas de disipación $p_{\text {dis }}$ :

$$
p_{\text {mov }}=p_{c}+p_{\text {dis }}
$$

Derivando la energía cinética requerida para conseguir el perfil de velocidad deseado, se obtiene $p_{c}$, definida por:

$$
p_{c}=m v \frac{d v}{d t}
$$

en donde $m$, es la masa total del vehículo y $v$, es la velocidad del perfil. Las fuerzas disipativas, son la fuerza aerodinámica, y la fuerza de fricción con el suelo. La suma de estas fuerzas, multiplicadas por el vector de velocidad $v$ forman $p_{d i s}$, y se define como:

$$
p_{d i s}=\frac{1}{2} \cdot p_{v} \cdot\left(S \cdot C_{x}\right) \cdot v^{3}+m \cdot g \cdot v \cdot C_{r r}+m \cdot g \cdot v \cdot \sin (\alpha)
$$

donde $g$ es la gravedad, $C_{r r}$ es el coeficiente de resistencia a rodamiento, $\alpha$ es la pendiente de la carretera, $p_{v}$ es la densidad del aire, $S$ es el área frontal del vehículo y $C_{x}$ es el coeficiente de arrastre aerodinámico. La Tabla IV muestra los parámetros usados para el vehículo.

TABLA IV

PARÁMETROS USADOS DEL VEHÍCULO

\begin{tabular}{lccc}
\hline \hline \multicolumn{1}{c}{ Nombre } & Símbolo & Valor & Unidad \\
\hline $\begin{array}{l}\text { Densidad del aire } \\
\text { Coeficiente de resistencia al } \\
\text { movimiento }\end{array}$ & $C_{r r}$ & 0.02 & {$\left[\mathrm{~kg} / \mathrm{m}^{3}\right]$} \\
$\begin{array}{l}\text { Coeficiente aerodinámico del } \\
\text { vehículo }\end{array}$ & $\mathrm{C}_{x}$ & 0.33 & {$\left[\mathrm{~kg} / \mathrm{m}^{3}\right]$} \\
$\begin{array}{l}\text { Área frontal del vehículo } \\
\text { Masa del vehículo }\end{array}$ & $S$ & 3.08 & {$\left[\mathrm{~kg} / \mathrm{m}^{3}\right]$} \\
Angulo de carretera & $m$ & 1200 & {$\left[\mathrm{~kg} / \mathrm{m}^{3}\right]$} \\
\hline \hline
\end{tabular}

\section{Bus de potencia}

Según se puede ver en la Fig. 1, el bus de potencia interconecta todos los componentes del sistema de gestión de energía del vehículo, pudiéndose establecer el siguiente balance:

$$
p_{\text {gen }}=p_{\text {fc }}+p_{\text {bat }}+p_{\text {sup }}+p_{\text {freno }}
$$

donde $p_{\text {freno }}$ es la potencia que se puede recuperar con el concepto de freno regenerativo y $p_{\text {gen }}$ es la potencia eléctrica total generada para el desplazamiento del vehículo. Para que se cumpla el perfil de velocidad, $p_{m o v}$ debe ser igual a $p_{g e n}$.

En la Fig. 3, se gráfica la ecuación (6), donde se muestra el balance de potencias. En línea continua, se muestra la potencia que es necesaria aportar para el cumplir el perfil de velocidad, y en línea discontinua, la potencia que puede ser recuperada mediante el concepto de frenado regenerativo.

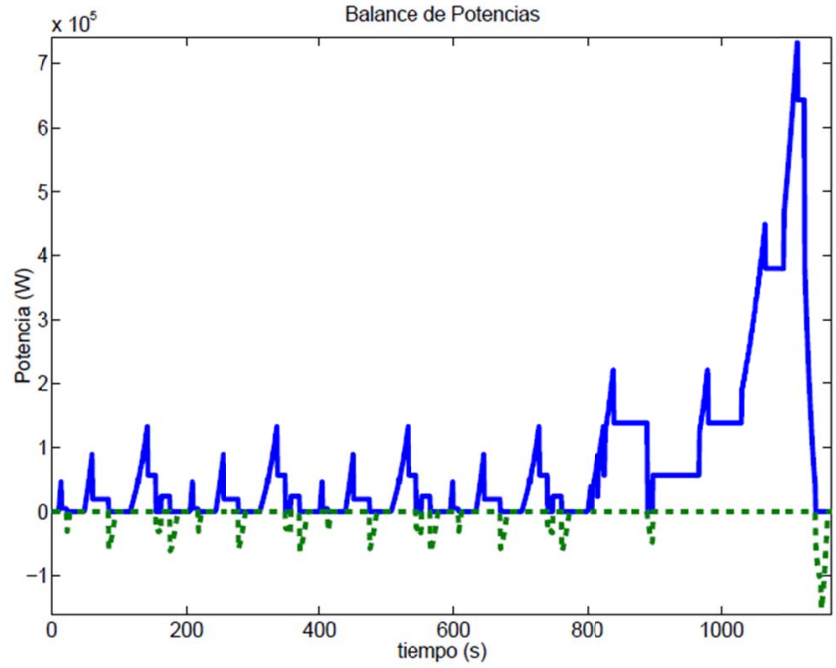

Fig. 3. Balance de potencias para el perfil NEDC.

\section{E. Frenado regenerativo}

El concepto de frenado regenerativo se traduce físicamente en un dispositivo que, al reducir la velocidad de un vehículo por el freno convencional, transforma una cantidad de energía cinética producida, en eléctrica. Esta energía se almacena según las limitaciones de los elementos disponibles para absorberla, [35]-[37].

El porcentaje máximo de energía recuperable \% $\%$ rec mediante el frenado regenerativo para un perfil de velocidad dado, se puede estimar a partir de la relación entre la energía recuperable $E_{\text {rec }}$, definida por:

$$
E_{\text {rec }}=\int_{0}^{t} p_{\text {rec }} d t
$$

Cuando $p_{\text {rec }}$ se define como, $p_{\text {rec }}=p_{c} \leq 0$. La energía requerida para el movimiento a la velocidad establecida por el perfil, viene dada por $E_{m o v}$, expresada como:

$$
E_{\text {mov }}=\int_{0}^{t} p_{\text {mov }} d t
$$


para $p_{c} \geq 0$. La relación entre las mismas, queda definida por:

$$
\% \text { rec }=\frac{E_{\text {rec }}}{E_{\text {mov }}}
$$

Para el vehículo considerado representado por los parámetros mostrados en la Tabla III y el perfil de velocidad NEDC, el máximo valor de recuperación se acerca al 13\%. En la Fig. 3, se puede observar en verde la potencia que se puede recuperar, la misma que viene de (6). Es importante recalcar que tan solo el $13 \%$ de la misma podrá ser recuperada.

\section{CONTROL PREDICTIVo ECONÓMICO (EMPC)}

El objetivo de la mayoría de los actuales sistemas de control avanzado es guiar al proceso a un punto de operación óptimo de forma rápida y fiable. Buscar el punto óptimo de referencia en estado estacionario se suele realizar por algún otro sistema de gestión de la información que determina, entre todos los estados estacionarios, cual es el más rentable [38]. Sin embargo, esta separación jerárquica de la información y del control no implica un funcionamiento óptimo especialmente en los transitorios. Una alternativa propuesta es tomar el objetivo económico directamente como parte de la función del sistema de control. Para que el controlador optimice directamente en tiempo real el desempeño económico del proceso, en lugar de seguimiento a un valor de consigna [39].

El control MPC se puede definir como una estrategia de control que se basa en un modelo matemático interno del proceso a controlar, más conocido como modelo de predicción. Dicho modelo se utiliza para predecir la evolución de las variables a controlar durante un intervalo de tiempo especificado. De este modo, se puede calcular las variables de control futuras " $u$ " para lograr que en dicho horizonte las salidas controlas " $y$ " converjan a sus respectivos valores de referencia. Esta optimización se lleva a cabo dentro de un horizonte de predicción, con el modelo inicializado al inicio del mismo a partir de las medidas (o estimación del estado del sistema). Dicha optimización se ejecuta en cada periodo de muestreo " $k$ " [40]. La señal de control a ser aplicada al proceso es obtenida resolviendo un problema de control óptimo de un criterio, función de coste (o función objetivo) en lazo abierto dentro de un horizonte de control finito y en cada periodo de muestreo $k$, sujeto a restricciones dadas por las características operativas del sistema, o por sus limitaciones físicas de sus componentes [41]. Como resultado de dicha optimización se obtiene una secuencia de señales de control óptimas en cada periodo de muestreo, de las cuales sólo la primera de ellas es aplicada al proceso y el horizonte de predicción se desplaza al siguiente instante de tiempo antes de iniciar la optimización nuevamente utilizando el principio de la ventana deslizante.

En el presente artículo, se traduce la parte económica de la función de coste como una herramienta para mejorar el consumo de combustible, es decir del hidrógeno, de manera que se traduzca en un ahorro del mismo.

A partir del modelo de los componentes del sistema de gestión de energía del HEV introducido en la sección anterior, se obtendrá el modelo de control en espacio de estados. Luego se incluirán las limitaciones de control y se definirán los objetivos del EMPC. El modelo a usar en este trabajo, tendrá en cuenta el nivel de gestión de energía de los componentes, y asumirá que existen controles locales de bajo nivel, que gestionan los niveles de referencia, corrientes, voltajes y demás variables particulares de cada elemento.

\section{A. Espacio de Estados}

El modelo en espacio de estado para la gestión de energía en un vehículo híbrido, se obtiene a partir las ecuaciones de los componentes elementales previa discretización en el tiempo:

$$
\begin{gathered}
x(k+1)=A x(k)+B u(k)+B_{p} d(k) \\
E_{u}(k)+E_{d} d(k)=0
\end{gathered}
$$

donde $x(k) \in \mathbb{R}^{n}$, es el vector de estados, que, para el caso en particular, representa la energía de la batería $E_{b a t}$, la energía del supercapacitor $E_{s u p}$, y la energía de pila de combustible $E_{f c}$; $u(k) \in \mathbb{R}^{m}$, es el vector de variables de control, que está compuesto por la potencia de la batería $P_{b a t}$, del supercapacitor $P_{\text {sup }}$, de la pila de combustible $P_{f c}$ y del freno regenerativo $p_{\text {freno }} ; d(k) \in \mathbb{R}^{p}$, es el vector que contiene las perturbaciones medidas en el sistema, que son las demandas de potencia $p_{\text {mov }}$ para seguir el perfil de velocidad. Nota: las demandas se han divido en dos partes, una primera demanda es la potencia necesaria para desplazar el vehículo $d^{+}$antes denominada $p_{\text {mov }}$ para para $p_{c} \geq 0$, y una segunda demanda es la energía que es capaz de recuperarse como parte del frenado regenerativo $d$ que anteriormente se la referenciaba como $p_{\text {rec }} ; A, B$ y $B_{p}$ son las matrices de estado, mientras $E$ y $E_{d}$ son matrices que describen la relación de equilibrio entre los estados, y llevan los signos de aporte de potencia $(+)$, o resta de potencia del sistema (-). Se debe entender que la pila de combustible, y las descargas de los elementos de almacenamiento, aportan al sistema, mientras las cargas de los mismos, piden energía (tomada del frenado).

\section{B. Restricciones de control y estado}

Las restricciones operativas de las señales de control se establecen mediante cotas:

$$
u_{\text {min }}(k) \leq u(k) \leq u_{\text {max }}(k)
$$

Para el caso de las restricciones de estado, podemos limitarlas como se muestra en:

$$
x_{\text {min }}(k) \leq x(k) \leq x_{\text {max }}(k)
$$

e incluirlas en el problema de optimización.

\section{Función de coste}

El principal objetivo de un sistema híbrido de energía renovable es aprovechar al máximo la energía disponible y satisfacer la demanda solicitada por las cargas, optimizando al mismo tiempo el almacenamiento o provisión de energía en exceso o déficit que esté presente para cada instante de tiempo. La función de coste es la traducción de los objetivos 
de control en una expresión matemática, la cual ha sido ampliamente estudiada en trabajos como [42] y [43]. En el control predictivo clásico se penalizan las desviaciones en las acciones de control futuras con respecto a la trayectoria de referencia definida convenientemente. En el control EMPC aplicado a sistemas de gestión de energía, la función de coste típicamente adopta la siguiente forma, según [44]:

$$
J(x, u)=\min \left[\sum_{k=0}^{H p-1} f_{1}(k)+\sum_{k=0}^{H p-1} f_{2}(k)+\sum_{k=0}^{H p-1} f_{3}(k)\right]
$$

Las funciones objetivo individuales en (17), de definen mediante:

1) El coste económico de operación:

$$
f_{1}(k)=\omega_{\alpha} \cdot \alpha \cdot u(k) \cdot \Delta(t)
$$

donde $\omega_{\alpha}$, es el peso que se le da en la función de coste al término económico y $\alpha$ es una matriz de pesos asociadas a cada una de las fuentes de energía, entendiéndose que las mismas son la $P_{b a t}, P_{\text {sup }}$ y $P_{f c}$.

2) El mantenimiento de umbrales de seguridad en los dispositivos de almacenamiento de energía:

$$
f_{2}(k)=\varepsilon(k)^{T} \cdot \omega_{x} \cdot \varepsilon(k)
$$

donde $\varepsilon(k)$ se define como el valor de penalización de las restricciones relacionadas con los valores de seguridad de los estados de carga de los elementos de almacenamiento de energía y $\omega_{x}$ es una matriz de pesos asociada a dichas penalizaciones.

3) La operación suave de las acciones de control:

$$
f_{3}(k)=\Delta u(k)^{T} \cdot \omega_{u} \cdot \Delta u(k)
$$

donde para obtener un control suave, definimos $\Delta u(k)$ como el vector de variaciones en la señal de control y $\omega_{u}$ la matriz de pesos asociada a las acciones de control. La formulación del problema de optimización y su resolución mediante programación cuadrática se realiza al usar CPLEX/TOMLAB como se muestra en [45], en donde se explican los detalles de su uso. Resumiendo, se trata entonces de tomar en cuenta las siguientes problemáticas, que se representan en el modelo de control:

a) El cumplimiento de las demandas del sistema, tanto para el aporte de energía, como para maximizar la fracción de energía recuperable mediante el frenado regenerativo.

b) Limitar la carga y descarga de los elementos de almacenamiento en las cotas establecidas.

c) Optimizar la energía aportada por la fuente principal (pila de combustible), para cumplir el perfil de velocidad dado.

La importancia de cada una de las funciones de peso dentro de la general, viene dada por los objetivos que se deseen alcanzar, ya sea el ahorro de combustible, la preservación de vida útil de los elementos, el evitar la operación indebida y el dimensionamiento de los mismos. Usualmente se llega a un compendio entre todos los mencionados con pesos ajustados en base a un análisis de Pareto. Para el caso del presente artículo, nos enfocaremos en el término económico, que se incluye en el control EMPC, el mismo que será orientado al ahorro de combustible.

\section{Control Multiobjetivo}

\section{A. Formulación}

Se introducirá brevemente un enfoque para resolver el problema de optimización multiobjetivo, en donde se buscará estudiar la variación de las compensaciones individuales de los objetivos de control, frente a la función total de coste. Entonces, un camino, es usar el método de la suma ponderada que permite que el problema de optimización multiobjetivo sea expresado mediante:

$$
\min _{y \in \mathbb{R}^{n}}\left[f_{1}(y), f_{2}(y), f_{3}(y), \ldots, f_{n}(y)\right]
$$

Para ello, se busca minimizar la función objetivo, construida como una suma de funciones objetivo individuales multiplicados por los coeficientes de ponderación. Estos coeficientes, son los que nos van a permitir definir la importancia que tomen cada una de las funciones como tales.

Esta minimización debe estar sujeta a:

$$
\begin{aligned}
& g(y) \geq 0 \\
& h(y) \geq 0
\end{aligned}
$$

en donde, " $y$ " representa las variables de decisión, que se corresponden con las secuencias de $[x, u]$. Entonces todas las $f_{i}$, se agrupan en un vector de coste:

$$
J(y)=\left[f_{1}(y), f_{2}(y), f_{3}(y), \ldots, f_{n}(y)\right]^{T}
$$

El vector $g$ y el vector $h$, representan las restricciones de igualdad y de desigualdad, denotadas por (15) y (16).

$$
\begin{gathered}
g(y)=\left[g_{1}(y), g_{2}(y), g_{3}(y), \ldots, g_{n}(y)\right]^{T} \\
h(y)=\left[h_{1}(y), h_{2}(y), h_{3}(y), \ldots, h_{n}(y)\right]^{T}
\end{gathered}
$$

Este método se ha utilizado ampliamente para proporcionar un único punto de solución que refleje las preferencias incorporados en la selección de un conjunto de pesos. Como diferentes funciones objetivo pueden tener diferente magnitud, la normalización de los objetivos es requerida para obtener una solución consistente de Pareto.

\section{B. Normalización}

Para aplicar el método de la frontera de Pareto, debemos introducir algunos conceptos. Deberemos buscar un minimizador $y^{i^{*}}$ para la función de coste individual $f_{i}(y)$.

Entonces los puntos de utopía, definidos por:

$$
J^{*}=\left[f_{1}^{*}, f_{2}^{*}, \ldots, f_{n f}^{*}\right]
$$

contienen los mínimos de las funciones objetivos individuales (18), (19) y (20), $f_{i}(y)^{i^{*}}$, además incluyen los mínimos individuales de los vectores de coste $f(y)^{i^{*}}$, el mismo que resulta el coste evaluado por el minimizador individual.

\section{Los puntos aproximados de Nadir}




$$
J^{N}=\left[f_{1}^{N}, f_{2}^{N}, \ldots, f_{n f}^{N}\right]^{T}
$$

contienen el peor valor para cada objetivo, obtenido a partir de los vectores de costes mínimos individuales $f_{i}^{N}=\max \left[\left(f_{i}\left(y_{j}{ }^{*}\right)\right]\right.$ con $j=1, \ldots, n_{f}$. Usando los minimizadores individuales, $f_{\alpha, i}=f(y)^{i *}$ como los denominados puntos de anclaje, la matriz de resultante $\theta$ contiene en ella la enésima columna del vector $f_{\alpha, i}-f^{*}$. Alternativamente, al usar los pseudo puntos de anclaje:

$$
f_{p \alpha, i}=\left[f_{1}^{N}, f_{2}^{N}, \ldots, f\left(y^{i^{*}}\right), \ldots, f_{n f}^{N}\right]^{T}
$$

La matriz de resultante $\theta$ tiene la enésima columna del vector $f_{p \alpha, i}-f^{*}$.

$$
\min _{y \in \mathbb{R}^{n}} J_{(y)}=\sum_{i=1}^{n f} \omega_{i} \cdot f_{i}(y)
$$

de donde $\omega$ es el vector de parámetros escalables, o denominados pesos, $f_{i}$ vendría a representar la ecuación (19), (20) o (21). Para los pesos tenemos:

$$
\sum_{i=1}^{n f} \omega_{i}=1, \omega_{i} \geq 0
$$

\section{Resultados OBTENIDOS}

La Fig. 4 muestra como se ha simulado el esquema de control del sistema de gestión de energía del vehículo propuesto en este artículo. El controlador EMPC recibe la potencia requerida para el movimiento tomando en cuenta el balance de potencias con las ecuaciones no lineales de la fuerza de resistencia al movimiento. Entonces, la demanda queda convertida en un punto de referencia de potencia, que viene dada por el perfil de velocidad aplicado al balance de fuerzas. De esta manera la parte no lineal del modelo del HEV considerado para la gestión de energía queda reducida a una demanda. Se muestra, además de que el controlador nos proporciona las estrategias de manejo óptimo de los flujos de potencia entre los diferentes elementos del sistema, teniendo en cuenta el estado de carga de los elementos de almacenamiento y la predicción de proporcionada por el modelo lineal utilizado por el EMPC. En una primera parte, se trabajará con un objetivo de control puramente económico, manteniendo los pesos $W_{u}$ y $W_{x}$ en cero, mientras que $W_{\alpha}$ es igual a 1. Para evaluar el controlador EMPC se han considerado inicialmente tres escenarios de simulación, con un horizonte de predicción de 10 muestras y un tiempo de muestreo de 1 segundo.

\section{A. Escenarios para la verificación de función de peso económica}

El primer escenario que se considera, asume que las fuentes de generación y almacenamiento que aportan al sistema presentan el mismo coste de generación. Cada valor de $\alpha$ para las potencias $P_{b a t}, P_{s u p}$ y $P_{f c}$ toma el valor de 0.5 . En el segundo escenario se considera que la pila de combustible sea una fuente con un coste de generación bajo, mientras que el aporte de las fuentes de almacenamiento al sistema, se las considera con costes de generación más elevados. El valor de $\alpha$ para $P_{f c}$ es de 0 , mientras para $P_{b a t} y P_{\text {sup }}$ toma el valor de 1 . En el tercer escenario, se considera que la pila de combustible es una fuente con un coste alto de generación, mientras que las demás fuentes poseen un coste menor al de la pila. El valor de $\alpha$ para $P_{f c}$ es de 1 , mientras para $P_{b a t} y P_{\text {sup }}$ toma el valor de 0 .

La Tabla $\mathrm{V}$, muestra la variación de la potencia entregada por la pila de combustible, dependiendo del escenario considerado. En dicha tabla, se comprueba que, penalizando el coste de la pila de combustible, se puede restringir el uso de la misma, o aumentar en caso de ser necesario.

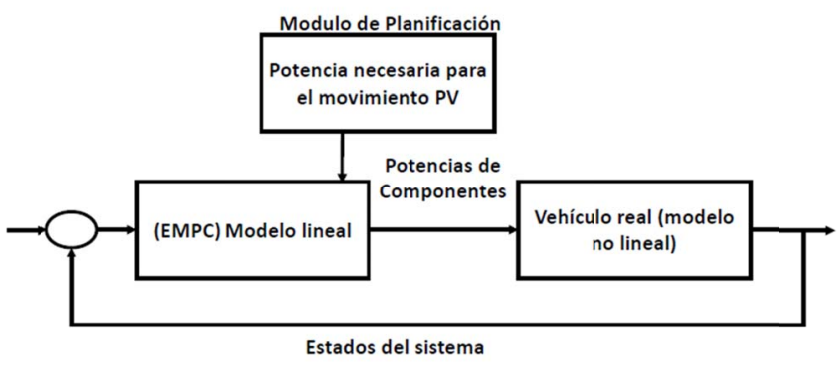

Fig. 4. Principio de funcionamiento del esquema de control.

TABLA V

APORTE DE LA PILA DE COMBUSTIBLE EN CADA ESCENARIO DE SIMULACIÓN

\begin{tabular}{lc}
\hline \multicolumn{1}{c}{ Coste de generación } & Energía entregada por la pila [J] \\
\hline Primer Escenario & $2,019,324.08$ \\
Segundo Escenario & $2,102,019.46$ \\
Tercer Escenario & $1,998,965.87$ \\
\hline
\end{tabular}

Dependiendo de los valores tomados por $\alpha$ dentro de la primera función de coste, mostrada en (18), podremos aumentar o disminuir el aporte y recuperación de las fuentes del sistema (baterías, supercapacitores y pila de combustible). Los valores mostrados en la Tabla $\mathrm{V}$, fluctúan dentro de un límite superior e inferior. Definiremos estos límites como la cantidad de energía máxima y mínima que la pila de combustible puede entregar. Entonces, el primer límite corresponde al caso en que la pila de combustible nos da la mayoría de energía del movimiento, debiéndose cumplir que:

$$
d^{+} \simeq p_{f c}
$$

Es decir, la pila de combustible debe generar el total de energía necesaria para cumplir el perfil de velocidad, pues queda como la única fuente de generación cuando las demás tienen un coste elevado y la función objetivo busca minimizar el consumo. El segundo límite, corresponde al caso en que la pila nos proporcione la menor cantidad de energía que le sea posible, sin dejar de cumplir el perfil, debiéndose cumplir que:

$$
d^{+}-\% d \simeq p_{f c}
$$

La Tabla VI y Tabla VII, nos muestran los valores de los límites obtenidos, observando que cumplen tanto (32) y (33).

El valor obtenido mediante (33) corresponde al caso donde se presenta el máximo uso del frenado de regenerativo. $\mathrm{Si}$ 
tomamos los valores de la energía obtenidos, y los introducimos en (12) para el HEV considerado, podremos observar que el resultado se acerca a un $13 \%$, con los que se comprueba, que los límites estén correctos. En este caso los elementos de almacenamiento recuperarán el máximo posible de energía del frenado. Es decir, si se recupera cerca de un $13 \%$ con el freno regenerativo, y al ser los estados de carga inicial y final iguales para los elementos de almacenamiento; si para el caso anterior la pila daba casi un $100 \%$ de la potencia necesaria, ahora debe dar cerca de un $87 \%(100 \%$ $13 \%$ ). Este valor, se acerca al $88,25 \%$ mostrado en la Tabla IV. La Fig. 5 y Fig. 6 muestran el flujo de potencia para el mencionado ajuste de pesos en el caso de máxima entrega de potencia de la pila de combustible.

TABLA VI

APORTE MÁXIMO DE LA PILA DE COMBUSTIBLE EN LA SIMULACIÓN

\begin{tabular}{ccc}
\hline \hline$E_{\text {mov }}[\mathrm{J}]$ & $E_{f c}[\mathrm{~J}]$ & $\%$ uso de la pila \\
\hline $2,177,473.40$ & $2,176,943.71$ & $99.88 \%$ \\
\hline \hline
\end{tabular}

TABLA VII

APORTE MÍNIMO SE LA PILA DE COMBUSTIBLE EN LA SIMULACIÓN

\begin{tabular}{ccc}
\hline \multicolumn{3}{c}{ APORTE MINIMO SE LA PILA DE COMBUSTIBLE EN LA SIMULACION } \\
\hline$E_{\text {mov }}-E_{\text {rec }}[\mathrm{J}]$ & $E_{f c}[\mathrm{~J}]$ & $\%$ uso de la pila \\
\hline $1,909,596.49$ & $1,921,803.5$ & $88.25 \%$ \\
\hline \hline
\end{tabular}
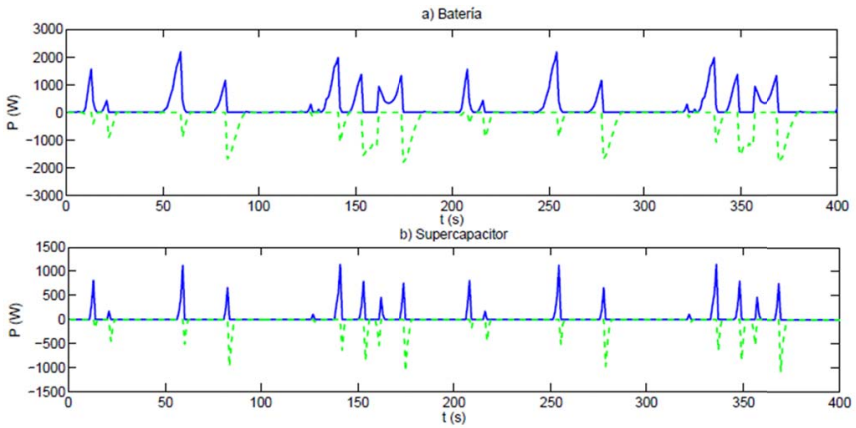

Fig. 5. Flujo de potencia de elementos de almacenamiento.
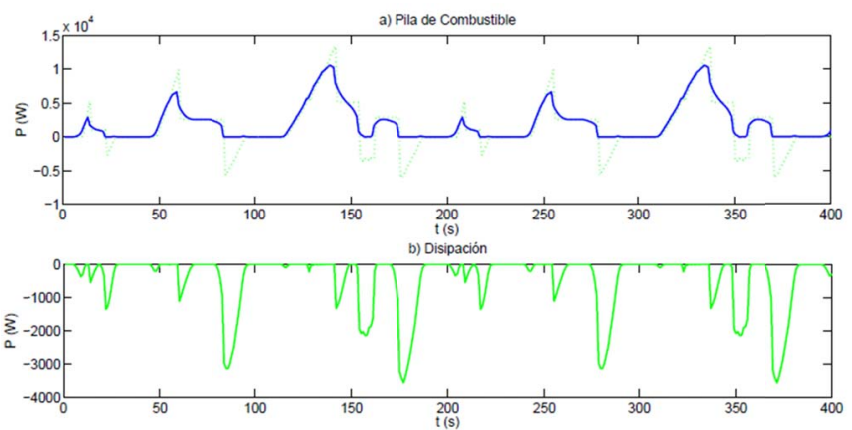

Fig. 6. Flujo de potencia de la pila y disipación.

En la Fig. 5, se ve la potencia del supercapacitor y de la batería. En línea continua azul, se muestra el aporte de potencia de la batería y supercapacitor respectivamente, mientras en la línea discontinua verde se ve la potencia recuperada del freno regenerativo por los elementos de almacenamiento. Esta figura muestra una parte de todo el perfil de velocidad NEDC de 400 muestras, para que se visualice mejor el balance de potencias.

En la Fig. 6, en la subgráfica 1, en línea continua azul, se observa la potencia entregada por la pila de combustible, y en línea entrecortada verde, la potencia total requerida para el movimiento $p_{m o v}$. Como se observa, los momentos en que la pila de combustible entrega energía, son en los momentos en las que el vehículo está en movimiento, mientras que cuando existen paradas deja de generar. En la subgráfica 2, se observa la disipación del freno mecánico, que es la energía que los elementos de almacenamiento no pueden recuperar. La Figura se muestra en el mismo espacio de tiempo que la anterior, es decir de 400 muestras del total del NEDC. Si sumamos la energía de la batería, la pila y el supercapacitor se debe cumplir la ecuación del balance energético. Esto se puede ver en parte gráficamente en las Fig. 5 y Fig. 6, pues al momento de necesitar energía para cumplir con el perfil de velocidad, los elementos la entregan. Del mismo modo, al existir el freno se recupera energía y la que no, se disipa.

\section{B. Ajuste de los pesajes}

Para el caso del control multiobjetivo, deberemos tomar en cuenta la función de coste completa, con cada uno de las funciones individuales mostradas en (18), (19) y (20). Una vez normalizado el sistema con las condiciones estudiadas en la Sección 4 , se varían los pesos $W_{u}, W_{x} y W_{\alpha}$ entre el $0 \%$ y $100 \%$ (0 a 1). La Fig. 7, muestra la compensación entre las contribuciones a la función de coste total, de las funciones individuales de coste económico de operación y de la operación suave de las acciones de control. La Fig. 8, muestra la compensación entre las contribuciones a la función de coste total, de las funciones individuales de coste económico de operación y el mantenimiento de umbrales de seguridad. Como se observa, mientras el valor de una función individual aumenta, el otro va decreciendo.

La Fig. 9 muestra en un gráfico tridimensional el compromiso que existe entre las funciones individuales ya mencionadas, manteniendo el mismo comportamiento que las figuras anteriores.

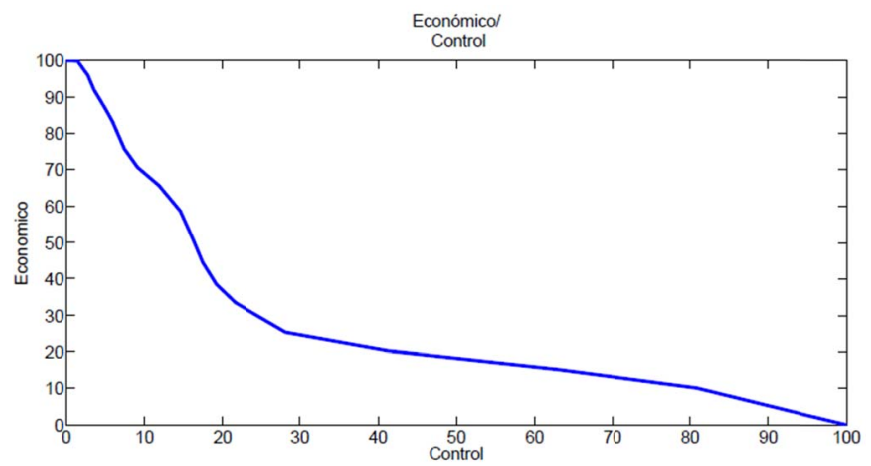

Fig. 7. Variación de la función de coste económico de operación versus la operación suave de las acciones de control. 


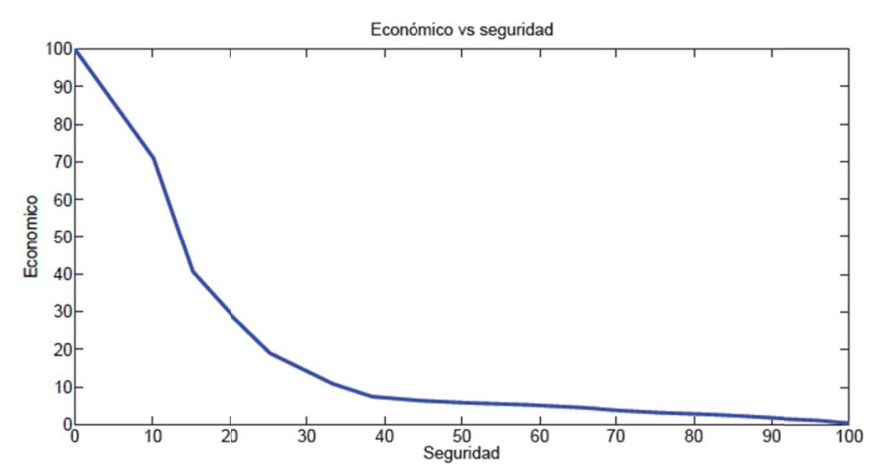

Fig. 8. Variación de la función de coste económico de operación versus mantenimiento de umbrales de seguridad.

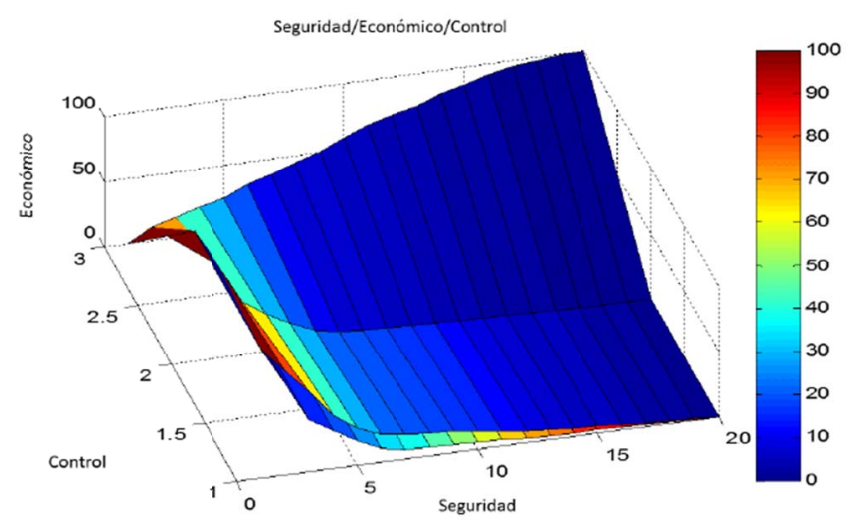

Fig. 9. Variación de la función económica vs seguridad vs la operación suave.

\section{CONCLUSIONES}

El control EMPC es una técnica de gestión óptima que permite incluir el coste operacional de los elementos que conforman el sistema directamente en la formulación de la función de coste. En el caso del presente artículo la potencia de la pila de combustible se relaciona con la cantidad o masa de hidrógeno consumida. Entonces, en la función de coste, además de los términos usados para los estados del sistema y las señales de control, el término económico puede ponderar el uso de las fuentes mediante pesos. El ajuste de los pesos, permite disminuir el uso de la pila de combustible desde su punto superior, en donde aporta cerca del $100 \%$ de la energía necesaria para el movimiento, hasta un límite inferior en donde aporta cerca del $88 \%$ de la energía necesaria, dando un margen de recuperación de cerca del 13\% de la energía del frenado. En el caso donde la pila de combustible genera menor cantidad de energía, se disminuyen también los picos de potencia que entrega, lo que ayuda a aumentar su tiempo de vida operacional y su eficiencia. La batería no absorbe los picos elevados de potencia, debido a la presencia del supercapacitor, el mismo que absorbe los picos grandes en momentos de tiempo más pequeños. También se presenta un enfoque inicial de control multiobjetivo, en donde se presentan las variaciones en las funciones de coste, según varía el pesaje sobre cada una de ellas. En este apartado se puede observar que mientras se aumenta el peso de una función de control, las siguientes van a disminuir en su magnitud. Por este motivo, dependiendo de las condiciones operacionales, y de lo que se desea lograr, se puede escoger el punto óptimo del tunning de los pesos.

Mediante técnicas de control óptimo como por ejemplo la programación dinámica (DP) se podrá para ciertos parámetros de una función de coste y operación de los elementos, conocer la mejor gestión energética. Es decir, la cantidad ideal que deberán aportar los elementos para cumplir con el mayor ahorro del combustible y alargar la vida útil de los mismos. Luego, en base a los parámetros escogidos, se podrá comparar las funciones de coste aplicando el control MPC y EMPC respectivamente. En futuros trabajo también se podrá hacer un control robusto, en el que se pongan cotas a los valores de potencia, de manera que el ciclo pueda considerar incertidumbre. También se podrá incluir el coste real en términos de consumo de Hidrógeno y del costo operativo de los elementos de almacenamiento, además del coste económico de los mismos, para llegar a un dimensionamiento óptimo que estará dentro de la curva presentada en este trabajo.

\section{AgRAdECIMIENTOS}

Este trabajo ha sido parcialmente financiado por las ayudas del Ministerio de Educación de España a través de los CICYT ECOCIS (ref. DPI2013-48243-C2-1-R), CICYT HARCRICS (ref. DPI2014-58104-R) y MICAPEM-IRI (DPI2015-69286C3-2-R), [MINECO/FEDER].

\section{REFERENCIAS}

[1] S. Alegre, J. V. Míguez, and J. Carpio, "Modelling of electric and parallel-hybrid electric vehicle using matlab/simulink environment and planning of charging stations through a geographic information system and genetic algorithms," Renewable and Sustainable Energy Reviews, vol. 74, pp. 1020-1027, 2017.

[2] J. Delbeke, G. Klaassen, and S. Vergote, "Climate-related energy policies," EU Climate Policy Explained, pp. 61-91, 2015.

[3] A. Nordlund, J. Jansson, and K. Westin, "New transportation technology: norm activation processes and the intention to switch to an electric/hybrid vehicle," Transportation Research Procedia, vol. 14, pp. 2527-2536, 2016.

[4] M. H. Ullah, T. Gunawan, M. Sharif, and R. Muhida, "Design of environmental friendly hybrid electric vehicle," in Proc. International Conference on Computer and Com- munication Engineering (ICCCE), 2012, pp. 544-548.

[5] L.-q. Jin, X.-h. Zeng, and W. Wang, "The control strategy and cost analysis for series plug-in hybrid electric vehicle," in Proc. 2nd International Conference on Advanced Computer Control (ICACC), 2010 , pp. 350-354.

[6] M. Hannan, F. Azidin, and A. Mohamed, "Hybrid electric vehicles and their challenges: A review," Renewable and Sustainable Energy Reviews, vol. 29, pp. 135-150, 2014.

[7] S. A. Rahman, N. Zhang, and J. Zhu, "A comparison on fuel economy and emissions for conventional hybrid electric vehicles and the uts plugin hybrid electric vehicle," in Proc. the 2nd International Conference on Computer and Automation Engineering (ICCAE), 2010, pp. 20-25.

[8] M. Ehsani, Y. Gao, and A. Emadi, Modern electric, hybrid electric, and fuel cell vehicles: fundamentals, theory, and design. CRC Press, 2009.

[9] A. Khaligh and Z. Li, "Battery, ultracapacitor, fuel cell, and hybrid energy storage systems for electric, hybrid electric, fuel cell, and plug-in hybrid electric vehicles: State of the art," Trans. on Vehicular Technology, vol. 59, no. 6, pp. 2806-2814, 2010. 
[10] S. Zhang and R. Xiong, "Adaptive energy management of a plug-in hybrid electric vehicle based on driving pattern recognition and dynamic programming," Applied Energy, vol. 155, pp. 68-78, 2015.

[11] C. Pan, L. Chen, L. Chen, C. Huang, and M. Xie, "Research on energy management of dual energy storage system based on the simulation of urban driving schedules," International Journal of Electrical Power \& Energy Systems, vol. 44, no. 1, pp. 37-42, 2013.

[12] J. Cao and A. Emadi, "A new battery/ultracapacitor hybrid energy storage system for electric, hybrid, and plug-in hybrid electric vehicles," Transactions on Power Electronics, vol. 27, no. 1, pp. 122-132, 2012.

[13] X. Liu, Q. Zhang, and C. Zhu, "Design of battery and ultracapacitor multiple energy storage in hybrid electric vehicle," in Proc. Vehicle Power and Propulsion Conference, 2009, pp. 1395-1398.

[14] S. M. Lukic, S. G. Wirasingha, F. Rodriguez, J. Cao, and A. Emadi, "Power management of an ultracapacitor/battery hybrid energy storage system in a hev," in Proc. Vehicle Power and Propulsion Conference, 2006, pp. 1-6.

[15] Z. Song, J. Li, X. Han, L. Xu, L. Lu, M. Ouyang, and H. Hofmann, "Multi-objective optimization of a semi-active battery/supercapacitor energy storage system for electric vehicles," Applied Energy, vol. 135, pp. 212-224, 2014.

[16] C.-C. Lin, H. Peng and J. Grizzle, "A stochastic control strategy for hybrid electric vehicles," in Proc. of the 2004 American Control Conference, 2004, pp. 4710-4715.

[17] C.-C. Lin, H. Peng, J. W. Grizzle, and J.-M. Kang, "Power management strategy for a parallel hybrid electric truck," Transactions on Contro Systems Technology, vol. 11, no. 6, pp. 839-849, 2003.

[18] R. Zhang and Y. Chen, "Control of hybrid dynamical systems for electric vehicles," in Porc. of the 2001 American Control Conference, 2001, pp. 2884-2889.

[19] V. H. Johnson, K. B. Wipke, and D. J. Rausen, "Hev control strategy for real-time optimization of fuel economy and emissions," SAE Technical Paper, Tech. Rep., 2000.

[20] P. Pisu, K. Koprubasi, and G. Rizzoni, "Energy management and drivability control problems for hybrid electric vehicles," in Proc. Of the 44th IEEE Conference on Decision and Control, 2005 and 2005 European Control Conference, 2005, pp. 1824-1830.

[21] P. Pisu and G. Rizzoni, "A supervisory control strategy for series hybrid electric vehicles with two energy storage systems," in Proc. of IEEE Conference Vehicle Power and Propulsion, 2005, pp. 8-14.

[22] M. Back, Pra"diktive Antriebsregelung zum energieoptimalen Betrieb von Hybridfahrzeugen. Univ.-Verlag Karlsruhe, 2005.

[23] V. Kumar, K. Rana, and P. Mishra, "Robust speed control of hybrid electric vehicle using fractional order fuzzy pd and pi controllers in cascade control loop," Journal of the Franklin Institute, vol. 353, no. 8, pp. 1713-1741, 2016.

[24] Z. Wei, J. Xu, and D. Halim, "Hev power management control strategy for urban driving," Applied Energy, vol. 194, pp. 705-714, 2017.

[25] S. Onori, L. Serrao, and G. Rizzoni, "Adaptive optimal supervisory control methods," in Hybrid Electric Vehicles. Springer, 2016, pp. 7987.

[26] C. Wu, Y. Hung, and C. Hong, "On-line supercapacitor dynamic models for energy conversion and management," Energy Conversion and Management, vol. 53, no. 1, pp. 337-345, 2012.

[27] R. Carter, A. Crude, and P. J. Hall, "Optimizing for efficiency or battery life in a battery/supercapacitor electric vehicle," Transactions on Vehicular Technology, vol. 61, no. 4, pp. 1526-1533, 2012.

[28] T. Fletcher, R. Thring, and M. Watkinson, “An Energy Management Strategy to concurrently optimise fuel consumption \& PEM fuel cell lifetime in a hybrid vehicle," International Journal of Hydrogen Energy, vol. 41, no. 46, pp. 21503-21515, Dec. 2016.

[29] M. Uzunoglu and M. Alam, "Dynamic modeling, design and simulation of a pem fuel cell/ultra-capacitor hybrid system for vehicular applications," Energy Conversion and Management, vol. 48, no. 5, pp. 1544 1553, 2007.

[30] L. Dubau, L. Castanheira, F. Maillard, M. Chatenet, O. Lottin, G. Maran- zana, J. Dillet, A. Lamibrac, J.-C. Perrin, E. Moukheiber et al., "A review of pem fuel cell durability: materials degradation, local heterogeneities of aging and possible mitigation strategies," Wiley Interdisciplinary Reviews: Energy and Environment, vol. 3, no. 6, pp. 540-560, 2014.
[31] G. Karavalakis, F. Alvanou, S. Stournas, and E. Bakeas, "Regulated and unregulated emissions of a light duty vehicle operated on diesel $/ \mathrm{palm}$ based methyl ester blends over nedc and a non-legislated driving cycle," Fuel, vol. 88, no. 6, pp. 1078-1085, 2009.

[32] S. F. Tie and C. W. Tan, "A review of energy sources and energy management system in electric vehicles," Renewable and sustainable energy reviews, vol. 20, pp. 82-102, 2013.

[33] A. Lidozzi and L. Solero, "Power balance control of multiple-input dcdc power converter for hybrid vehicles," in Proc. of the 2004 IEEE International Symposium on Industrial Electronics, 2004, pp. 1467- 1472.

[34] S. Campanari, G. Manzolini, and F. G. De la Iglesia, "Energy analysis of electric vehicles using batteries or fuel cells through well-to-wheel driving cycle simulations," Journal of Power Sources, vol. 186, no. 2, pp. 464-477, 2009.

[35] L. Li, X. Li, X. Wang, J. Song, K. He, and C. Li, "Analysis of downshifts improvement to energy efficiency of an electric vehicle during regenerative braking," Applied Energy, vol. 176, pp. 125-137, 2016.

[36] C. Qiu and G. Wang, "New evaluation methodology of regenerative braking contribution to energy efficiency improvement of electric vehicles," Energy Conversion and Management, vol. 119, pp. 389-398, 2016.

[37] P. Xiao, J. Lou, L. Niu, and H. Gao, "Modeling and simulation of regenerative braking performance of electric vehicles based on decoupling strategy.” Key Engineering Materials, vol. 693, 2016.

[38] J. L. Rosero Beltra'n, "Estrategia de control de modelo predictivo para el despacho y almacenamiento de energ' 1 a renovable en sistemas h'ibridos," Master's thesis, Monterrey: Instituto Tecnolo'gico y de Estudios Superiores Monterrey, 2012.

[39] J. B. Rawlings, D. Angela, and C. N. Bates, "Fundamentals of economic model predictive control," in Proc. of the 51st IEEE conference on decision and control (CDC), 2012, pp. 3851-3861.

[40] T. Hofman, R. van Druten, M. Steinbuch, and A. Serrarens, "Rule-based equivalent fuel consumption minimization strategies for hybrid vehicles," IFAC Proceedings Volumes, vol. 41, no. 2, pp. 5652-5657, 2008.

[41] B.-C. Chen, Y.-Y. Wu and H.-C. Tsai, "Design and analysis of power management strategy for range extended electric vehicle using dynamic programming," Applied Energy, vol. 113, pp. 1764-1774, 2014.

[42] R. Amrit, J. B. Rawlings, and L. T. Biegler, "Optimizing process economics online using model predictive control," Computers \& Chemical Engineering, vol. 58, pp. 334-343, 2013.

[43] B. P. Omell and D. J. Chmielewski, "Igcc power plant dispatch using infinite-horizon economic model predictive control," Industrial \& Engineering Chemistry Research, vol. 52, no. 9, pp. 3151-3164, 2013.

[44] M. Ellis, H. Durand, and P. D. Christofides, "A tutorial review of economic model predictive control methods," Journal of Process Control, vol. 24, no. 8, pp. 1156-1178, 2014.

[45] R. Toro Olmedo, "Smart tuning of predictive controllers for drinking water networked systems," Master's thesis, Universitat Politécnica de Catalunya, 2010. 